\title{
Capacités discriminantes et caractère prédictif d'une épreuve de type "épreuves classantes nationales » en France
}

\section{Discriminant ability and predictive characteristics of a type National Ranking examination in France}

\author{
Rachel VIEUX ${ }^{1}$, Yannick BEJOT ${ }^{2}$, Marc BRAUN ${ }^{3}$, et François KOHLER ${ }^{4}$ \\ ${ }^{1}$ Service de Réanimation Néonatale, Soins Intensifs et Médecine Néonatale, Maternité Régionale Universitaire, \\ 54000 Nancy, France \\ 2 Service de Neurologie, CHU le Bocage, 21000 Dijon, France \\ 3 Conseil Pédagogique, Faculté de Médecine, Université Henri-Poincaré Nancy-1, 54000 Nancy, France \\ 4 Laboratoire Santé Publique Information médicale et Enseignement Assisté par Ordinateur (SPI-EAO), \\ Université Henri Poincaré Nancy-1, 54000 Nancy, France
}

Manuscrit reçu le 11 juillet 2011 ; commentaires éditoriaux formulés aux auteurs le 22 août et le 10 octobre 2011 ; accepté pour publication le 17 octobre 2011

Mots-clés Épreuves classantes nationales ; reproductibilité ; pouvoir discriminant
Résumé - Contexte : Le rang de classement aux épreuves classantes nationales (ECN) permet aux étudiants en médecine de choisir la spécialité de leur exercice futur et la ville de formation. Des épreuves blanches les préparent à ces épreuves. But : Évaluer les capacités discriminantes et le caractère prédictif d'une épreuve blanche type ECN. Matériel et méthodes : Les capacités discriminantes des dix dossiers de l'épreuve blanche type ECN de la grande région Nord-Est ont été déterminées à partir de leur étendue de rangs de classement et de leur influence propre sur le rang de classement de chaque étudiant. Nous avons établi une corrélation entre le rang de classement à l'épreuve blanche type ECN de la grande région Nord-Est pour les étudiants de Nancy et leur rang de classement aux ECN de juin 2010, ainsi qu'une corrélation entre les résultats aux ECN et les scores obtenus aux modules du deuxième cycle des études médicales (DCEM). Résultats : Les scores à l'épreuve blanche type ECN des 211 étudiants nancéens ont été recueillis. Aucun dossier de ces ECN blanches n'était discriminant et la corrélation avec les ECN 2010 était médiocre $\left(R^{2}=4 \times 10^{-4}\right.$ pour les étudiants les mieux classés à l'épreuve blanche). Le rang de classement aux ECN n'était expliqué ni par le rang de classement à l'épreuve blanche type ECN, ni par les scores obtenus par les étudiants au cours de leur deuxième cycle des études médicales. Conclusion : L'épreuve blanche type ECN de la grande région Nord-Est ne permettait pas de prédire le classement aux épreuves type ECN. 


$\begin{array}{ll}\text { Keywords } & \text { Résumé - Background: The ranking for the French National Ranking (NR) allows medical } \\ \text { National ranking; } & \text { students to choose a specialty for their future medical career. Practice examinations are designed } \\ \text { reproducibility; } & \text { to help them prepare for the NR. Aim: To assess the discriminant ability and the predictive char- } \\ \text { discriminant ability } & \text { acteristics of a practice examination. Methods: The discriminant ability of all questions of a } \\ \text { practice examination in the North East of France was determined. The ranking of all Nancy stu- } & \text { dents at the practice examination was measured and compared with that obtained by the same } \\ & \text { students at the } 2010 \text { NR to determine the correlation between the practice examination and the } \\ & \text { real competitive examination. Results: The scores of } 211 \text { students were analyzed. The practice } \\ & \text { examination did not identify any discriminant criteria. There was a weak correlation between } \\ & \text { the practice examination and the NR. The correlation for the top-ranked students at the practice } \\ & \text { examination was } R^{2}=4 \times 10^{-4} \text {. The ranking obtained at } 2010 \text { NR could neither be explained } \\ & \text { by students' performance during their academic courses or their ranking at the practice exam- } \\ & \text { ination. Discussion and conclusion: The practice examination did not provide adequate dis- } \\ & \text { criminant abilities and unsuccessfully predicted national ranking. }\end{array}$

\section{Introduction}

La réforme des études médicales a conduit à la mise en place en 2004 d'épreuves classantes nationales $(E C N)$. Depuis cette date, l'ensemble des étudiants en médecine ont l'obligation de se présenter à ces ECN à l'issue du deuxième cycle des études médicales, c'est-à-dire à la fin de leur cursus «pré-gradué ».

De 2004 à 2008, ces ECN reposaient sur l' analyse par les étudiants de neuf dossiers cliniques comportant chacun quatre à 10 questions explorant les champs du diagnostic et de la thérapeutique, avec un temps d'épreuve d'une heure par dossier. À compter de 2009, une épreuve supplémentaire, portant sur la lecture critique d'article (LCA), a été introduite avec l'objectif de former les futurs médecins à la démarche de la médecine fondée sur les preuves (evidencebased medecine $)^{[1]}$, nécessaire à l'analyse critique des nouvelles informations scientifiques reçues au cours d'une carrière médicale. Cette épreuve, d'une durée de trois heures, comporte d'une part quatre à 10 questions rédactionnelles relatives à un article médical original en français et, d'autre part, la rédaction d'un résumé structuré de 250 mots maximum. Désormais, elle compte pour $10 \%$ du score final des ECN.

À l'issue des ECN, les étudiants sont classés en fonction du score obtenu. Ils choisissent alors, par rang de classement croissant, leur centre hospitalier universitaire de rattachement et leur filière de formation spécialisée du troisième cycle (cursus «postgradué ») parmi les suivantes : anesthésie-réanimation, gynécologie-obstétrique, médecine générale, pédiatrie, psychiatrie, médecine du travail, santé publique, spécialités médicales, spécialités chirurgicales. De ce fait, le classement aux ECN représente un enjeu personnel important car il détermine la suite de la carrière des futurs médecins. Dans la plupart des facultés, les étudiants s'y préparent et se testent à l'occasion d'épreuves d'entrainement dites «ECN blanches ».

Dès leur mise en place, et au fur et à mesure de leur application, les ECN ont suscité un vif débat au sein de la communauté pédagogique médicale ${ }^{[2-6]}$ et l'introduction de la LCA n'a fait qu' ajouter à ce débat tant les modalités de celle-ci ont été contestées ${ }^{[7-12]}$. Un certain nombre de points faibles en ont été soulignés : ces épreuves n'ont pas de valeur certifiante puisqu'il n'est pas requis d'obtenir un score minimal pour accéder au choix de filière de formation spécialisée du troisième cycle. En ce sens, les ECN ne peuvent donc pas être considérées comme un examen ${ }^{[5,13]}$. Leur objectif est de classer les étudiants, ce qui, en matière psychométrique, nécessite d'optimiser le caractère discriminant des épreuves en rendant plus tangible les écarts de scores entre les candidats ${ }^{[5]}$. Cette fonction classante (évaluation à interprétation normative) nécessiterait en toute rigueur que les ECN satisfassent scrupuleusement les 
critères de qualité métrologique. Pourtant, certains auteurs ont suggéré que les caractéristiques docimologiques des épreuves n'offraient pas un pouvoir discriminant suffisant et seraient probablement associées à une fidélité médiocre, notamment du fait de leur caractère réducteur lié à l'utilisation d'un seul outil d'évaluation $^{[2]}$.

L'objectif de ce travail était: 1) d'évaluer les qualités discriminantes des dossiers d'une épreuve blanche de type ECN et 2) d'évaluer s'il était possible aux étudiants de prédire leur classement aux ECN officielles sur la base du classement obtenu aux ECN blanches.

\section{Matériel et méthodes}

\section{Type d'étude et données}

Nous avons mené une étude mono-centrique portant sur les résultats des étudiants de la Faculté de médecine de Nancy enregistrés à l'épreuve blanche de type ECN soumise en janvier 2010 aux étudiants de quatrième année du deuxième cycle des études médicales (DCEM4) des facultés de médecine de la région Grand-Est : Besançon, Dijon, Nancy, Reims et Strasbourg. Cette épreuve blanche comportait, comme les ECN officielles, un ensemble de dix dossiers dont neuf dossiers cliniques et un dossier de LCA, l'ensemble noté sur 1000 points, à raison de 100 points par dossier. Cette épreuve blanche avait pour objectif de réaliser une évaluation des étudiants d'une inter-région quelques mois avant les ECN officielles, avec le rendu d'un premier classement des étudiants de cette inter-région, avant le classement final et définitif des ECN de juin de la même année.

Les dossiers soumis aux étudiants lors de l'épreuve blanche répondaient aux recommandations d'écriture de dossiers de type ECN et leur contenu correspondait aux items à acquérir dans le cadre de la préparation des ECN. Chaque dossier de l'épreuve blanche de l'inter-région Grand-Est de 2010 avait été validé par un bureau de docimologie local appartenant à l'Université et spécialisé dans la docimologie appliquée aux études médicales, constitué d'experts en docimologie. Ces dossiers avaient ensuite été validés par des experts de l'inter-région. Les grilles de correction établies pour les dossiers de l'épreuve blanche avaient été évaluées par des professeurs des universités non spécialistes du thème du dossier de l'épreuve blanche, afin d' affiner au mieux la précision de ces grilles.

La base de données pour notre analyse comportait les scores obtenus aux dix dossiers par les étudiants de la faculté de médecine de Nancy. Cette base de données comportait également : les scores attribués à chaque étudiant pour chacun des dossiers par les deux correcteurs (le score de chaque dossier étant la moyenne des scores délivrés par les correcteurs 1 et 2) ; le score issu de la troisième correction si l'écart de score entre les deux correcteurs était supérieur à 15 (dans ce cas, le score de cette troisième correction était attribué au dossier) et le score final de chaque étudiant à cette épreuve blanche (somme des scores des dix dossiers).

Le bureau de docimologie de la faculté de Médecine Nancy-1 avait donné son accord pour l'analyse de cette base de données qui a été anonymisée après fusion avec les résultats des ECN officielles publiés sur internet.

\section{Analyse statistique}

Nous avons déterminé pour chaque étudiant le rang de classement à l'épreuve blanche de type ECN en fonction de son score final. Le score obtenu à chaque dossier de l'épreuve blanche a été analysé, ainsi que son influence sur le rang de classement. Les résultats de chaque dossier sont présentés par la médiane et l'étendue, les scores obtenus aux différents dossiers n'ayant pas une distribution gaussienne.

Un dossier discriminant a été défini comme un dossier dont l'étendue des scores était large, permettant ainsi d'établir aisément la distinction entre bons et mauvais résultats des étudiants. Nous avons établi une pondération des scores obtenus à l'épreuve blanche en fonction de l'espace interquartile de distribution des scores pour chaque dossier. Le score final était doté d'un coefficient égal au dixième de l'espace inter-quartile, afin d'accroître le poids des dossiers les plus discriminants dans le score final. 
Un dossier discriminant était également défini comme un dossier dont le score influençait de façon importante le rang de classement des étudiants.

Enfin, nous avons établi une corrélation par le coefficient de corrélation de Pearson entre le classement à l'épreuve blanche de type ECN et le classement aux ECN officielles. Nous avons également recherché la corrélation entre les scores obtenus aux modules du deuxième cycle et les scores des épreuves de type ECN. Pour cela, la moyenne des scores obtenus aux modules du deuxième cycle des études médicales (quatrième, cinquième et sixième année des études de médecine) a été calculée sur la base des scores obtenus à la première session de chaque examen.

\section{Résultats}

\section{Descriptif de l'épreuve blanche de type ECN}

L'épreuve blanche de type ECN comportait dix dossiers, qui portaient sur les thèmes suivants : psychiatrie $(n=1)$, gastro-entérologie $(n=1)$, neurologie $(n=1)$, endocrinologie $(n=1)$, pneumologie $(n=1)$, pédiatrie $(n=2)$, gériatrie $(n=1)$, toxicologie $(n=1)$, LCA $(n=1)$. Deux cent onze étudiants en DCEM4 de Nancy ont composé à l'occasion de cet examen. Seuls les dossiers 1 à 3 ont été rédigés par l'ensemble des étudiants. Tous les étudiants ont rédigé les dossiers 1 à 3 . Deux $(0,9 \%)$ à $3(1,4 \%)$ étudiants n'ont pas rédigé les dossiers 4 à 10 .

La distribution des scores finaux n'avait pas une étendue large (figure 1a). Même si les scores s'étendaient de 81,5 à 706, $50 \%$ des étudiants avaient un score final compris entre 531,5 (Q1) et 624,5 (Q3). La distribution des scores à chaque dossier est présentée dans le tableau I.

Le recours à une troisième correction a été nécessaire pour tous les dossiers, sauf pour le dossier 8 (tableau I). Pour les dossiers 3 à 7 et 10 , cette troisième correction a été requise pour plus de $9 \%$ des dossiers individuels corrigés, avec un maximum atteint par le dossier 3 ( $n=34$ troisièmes corrections, soit $16 \%$ des dossiers) avec un écart de scores entre
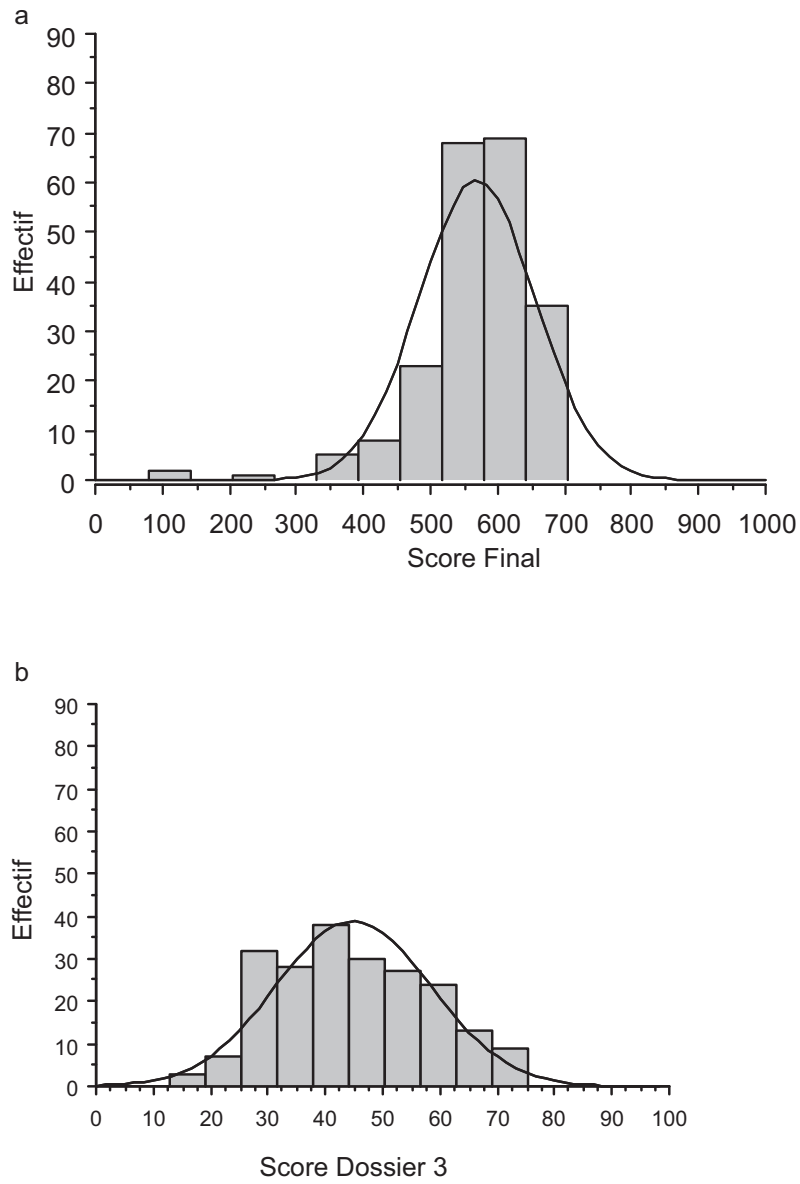

Fig.1. a) Histogramme des scores finaux des étudiants de DCEM4 de Nancy à l'épreuve blanche de type ECN ; b) scores obtenus au dossier 3 de l'épreuve blanche type ECN.

DCEM4 : Deuxième Cycle des Études Médicales, quatrième année ; ECN : Épreuves Classantes Nationales.

les deux correcteurs s'élevant à 49 (dossier noté sur 100 points).

\section{Qualités discriminantes des dossiers}

Les dossiers 1, 4 et 5 avaient l'étendue de scores maximale. Cependant, le dossier 3 avait une distribution qui semblait graphiquement la plus discriminante (figure 1b). Les dossiers 3 et 9 avaient la plus large étendue de rang de classement, c'est-à-dire le moins d'ex aequo (tableau II). 
Tableau I. Distribution des scores aux dix dossiers de l'épreuve blanche de type ECN.

\begin{tabular}{lllllllc} 
& & Médiane & Minimum & Maximum & Étendue & $\begin{array}{c}\mathbf{3}^{\mathbf{e}} \\
\text { correction }\end{array}$ & $\begin{array}{c}\text { Écart maximal de } \\
\text { scores entre 2 } \\
\text { correcteurs }\end{array}$ \\
\hline D1 & $n=211$ & 64,5 & 24,0 & 95,5 & 71,5 & $n=7$ & 33 \\
\hline D2 & $n=211$ & 70,5 & 25,0 & 91,5 & 66,5 & $n=9$ & 30 \\
\hline D3 & $n=211$ & 44,0 & 13,0 & 75,5 & 62,5 & $n=34$ & 49 \\
\hline D4 & $n=209$ & 58,5 & 12,0 & 84,0 & 72,0 & $n=19$ & 26 \\
\hline D5 & $n=209$ & 44,0 & 10,0 & 82,0 & 72,0 & $n=19$ & 25 \\
\hline D6 & $n=208$ & 67,0 & 25,5 & 82,0 & 56,5 & $n=21$ & 26 \\
\hline D7 & $n=209$ & 51,5 & 17,0 & 79,5 & 62,5 & $n=21$ & 26 \\
\hline D8 & $n=208$ & 68,0 & 28,5 & 90,0 & 61,5 & $n=0$ & 0 \\
\hline D9 & $n=208$ & 63,5 & 31,0 & 90,0 & 59,0 & $n=17$ & 44 \\
\hline D10 & $n=209$ & 51,5 & 22,0 & 76,5 & 54,5 & $n=22$ & 29 \\
\hline
\end{tabular}

D : Dossier ; ECN : Épreuves Classantes Nationales. Chaque dossier est noté sur 100.

Tableau II. Effet de chaque dossier sur le rang de classement final à l'épreuve blanche de type ECN.

\begin{tabular}{|c|c|c|c|c|}
\hline & \multirow{2}{*}{$\begin{array}{l}\text { Étendue du rang de } \\
\text { classement } \\
\text { par dossier }\end{array}$} & \multicolumn{3}{|c|}{$\begin{array}{c}\text { Effet du retrait du dossier sur le rang de classement à l'épreuve } \\
\text { blanche type ECN }\end{array}$} \\
\hline & & Médiane & Minimum & Maximum \\
\hline Dossier 1 & 81 & 2 & -2 & 6 \\
\hline Dossier 2 & 69 & -11 & -2 & -6 \\
\hline Dossier 3 & 93 & 2 & -2 & 5 \\
\hline Dossier 4 & 81 & -2 & -2 & -1 \\
\hline Dossier 5 & 83 & -1 & -2 & 4 \\
\hline Dossier 6 & 73 & 1 & -2 & 8 \\
\hline Dossier 7 & 83 & -1 & -2 & 2 \\
\hline Dossier 8 & 79 & 2 & -2 & 2 \\
\hline Dossier 9 & 96 & -1 & -2 & 4 \\
\hline Dossier 10 & 69 & -4 & -2 & 0 \\
\hline
\end{tabular}

ECN : Épreuves Classantes Nationales.

La pondération du score de chaque dossier par le dixième de l'espace interquartile des scores obtenus pour ce dossier a permis de calculer un score final pondéré et d'établir un nouveau rang de classement en fonction de ce score final pondéré sur un paramètre de dispersion. Ce nouveau rang de classement est présenté sur la figure 2 .
Le rang de classement était en général peu modifié par chaque dossier retiré individuellement. La différence entre le rang de classement final à l'épreuve blanche de type ECN et le rang de classement tel qu'il aurait été si tel ou tel dossier avait été retiré tour à tour du score final est présentée dans le tableau II. Le dossier 2 influençait le plus le rang de classement avec 


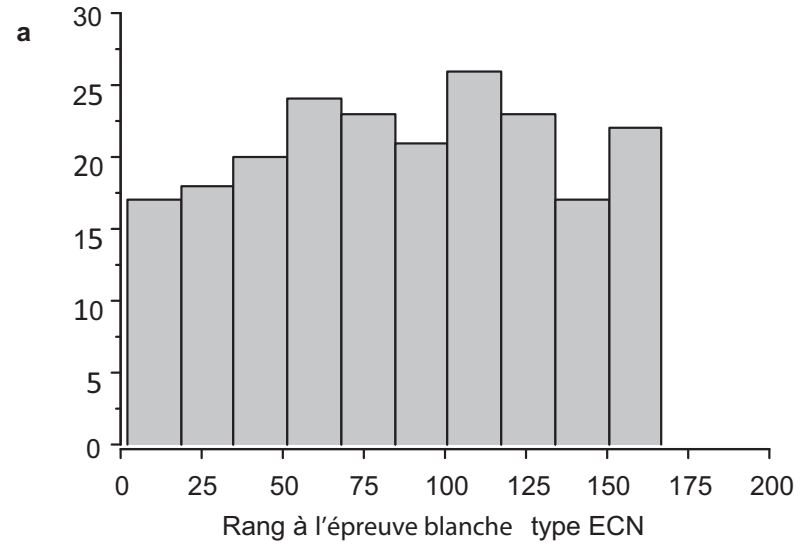

b

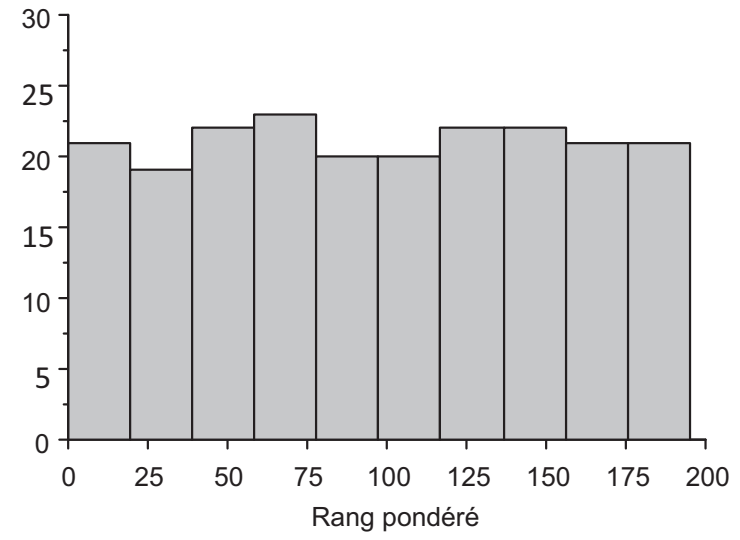

Fig.2. Distribution des rangs de classement; a) rang de classement en fonction du score final obtenu à l'épreuve blanche de type ECN ; b) rang de classement en fonction du score final pondéré pour chaque dossier. ECN : Épreuves Classantes Nationales.

une médiane de rang de classement diminuée de 11 places en cas de soustraction du score de ce dossier du score final. Les autres dossiers qui influençaient le plus le classement final étaient le dossier 1 ( +6 places au rang de classement final en cas de retrait de ce dossier) et le dossier 6 ( +8 places au rang de classement final en cas de retrait de ce dossier).

\section{Corrélation entre l'épreuve blanche de type ECN et les ECN officielles}

La corrélation entre l'épreuve blanche de type ECN et les ECN officielles était mauvaise avec une dispersion importante des points sur le nuage de points (figure 3). Un tableau récapitulatif du rang de

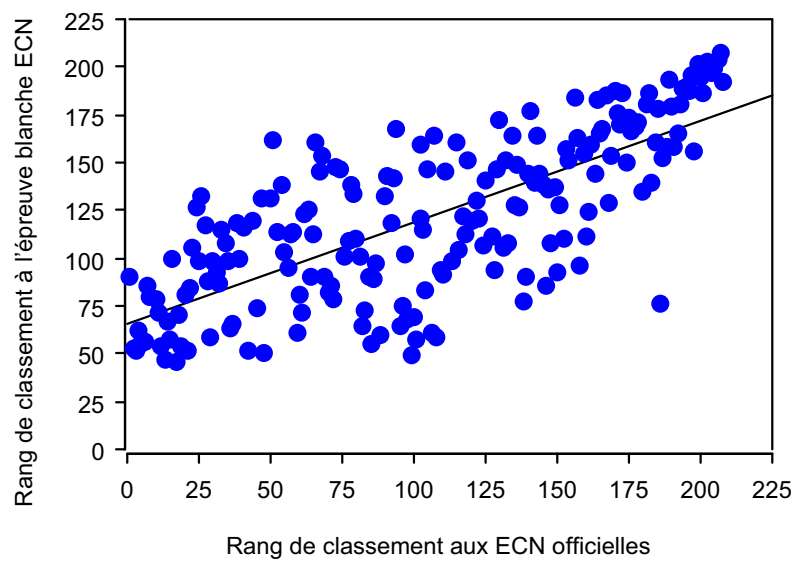

Fig.3. Corrélation entre le rang de classement aux ECN officielles et le rang de classement à l'épreuve blanche de type ECN. ECN : Épreuves Classantes Nationales.

Tableau III. Rang de classement aux ECN officielles et à l'épreuve blanche de type ECN pour 39 étudiants nancéens.

\begin{tabular}{|c|c|c|c|}
\hline Rang ECN & Rang blanc & Rang ECN & Rang blanc \\
\hline 1 & 90 & 21 & 51 \\
\hline 2 & 53 & 22 & 84 \\
\hline 3 & 51 & 23 & 105 \\
\hline 4 & 62 & 24 & 126 \\
\hline 5 & 55 & 25 & 99 \\
\hline 6 & 56 & 26 & 133 \\
\hline 7 & 86 & 27 & 117 \\
\hline 8 & 80 & 28 & 88 \\
\hline 10 & 78 & 29 & 59 \\
\hline 11 & 72 & 30 & 98 \\
\hline 12 & 54 & 31 & 92 \\
\hline 13 & 47 & 32 & 87 \\
\hline 14 & 67 & 33 & 115 \\
\hline 15 & 57 & 34 & 108 \\
\hline 16 & 100 & 35 & 99 \\
\hline 17 & 46 & 36 & 63 \\
\hline 18 & 70 & 37 & 66 \\
\hline 19 & 54 & 38 & 118 \\
\hline 20 & 81 & 39 & 100 \\
\hline
\end{tabular}

ECN : Épreuves Classantes Nationales.

classement obtenu à l'épreuve blanche par les quarante étudiants de Nancy les mieux classés à l'ECN est présenté à titre indicatif sur le tableau III. 
La corrélation entre les classements respectifs à l'épreuve blanche de type ECN et aux ECN officielles était mauvaise, en particulier pour les étudiants ayant obtenu de bons résultats à l'épreuve blanche, pour lesquels il n'y avait pas de corrélation entre le classement aux ECN officielles et classement à l'épreuve blanche $\left(R^{2}=4 \times 10^{-4}\right)$.

Scores obtenus aux modules du deuxième cycle et rang de classement aux épreuves de type ECN

La corrélation entre les scores obtenus aux ECN officielles ou à l'épreuve blanche et le score moyen obtenu aux modules validants du deuxième cycle était respectivement $R^{2}=0,45$ et $R^{2}=0,40$. Le nuage de points est présenté sur la figure 4 .

\section{Discussion}

Cette étude objective d'une part que les dossiers proposés à l'épreuve blanche de type ECN de l'interrégion Grand-Est de janvier 2010 n'avaient pas de qualité discriminante et, d'autre part, que les résultats de ces épreuves et des examens de deuxième cycle prédisaient mal le rang de classement aux ECN officielles à l'échelon individuel.

Pouvoir discriminant des dossiers de l'épreuve blanche de type ECN de l'inter-région Grand-Est de janvier 2010

Aucun dossier de l'épreuve blanche ne présentait une étendue large de scores des étudiants. Certains dossiers (dossiers 1, 4 et 5) avaient une étendue de scores supérieure à 70 ; cependant, même pour ces dossiers, la plupart des étudiants avaient un score proche de la médiane. Ceci implique, sur un effectif plus grand comme celui des candidats aux ECN (environ 7000 en 2010), un risque élevé d'égalité de scores et, par conséquent, une difficulté à classer les étudiants.

L'analyse des rangs de classement par dossier conforte ces résultats. L'étendue des rangs de

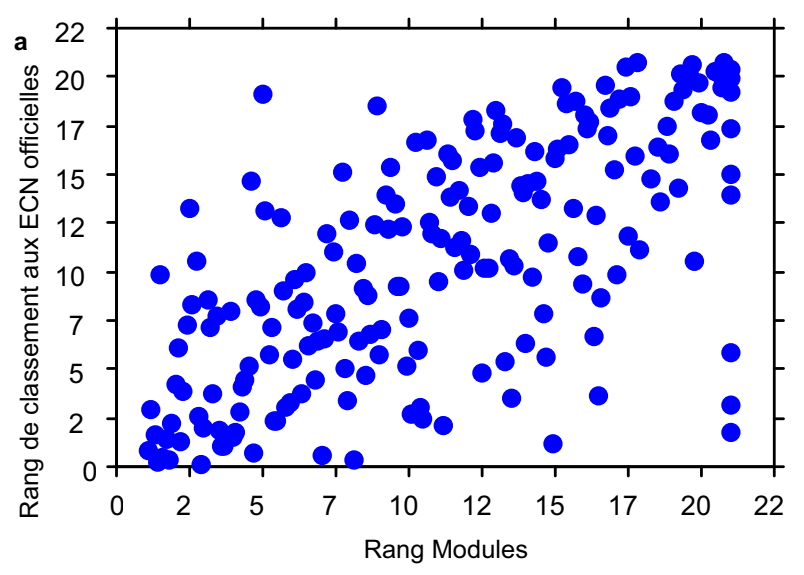

b

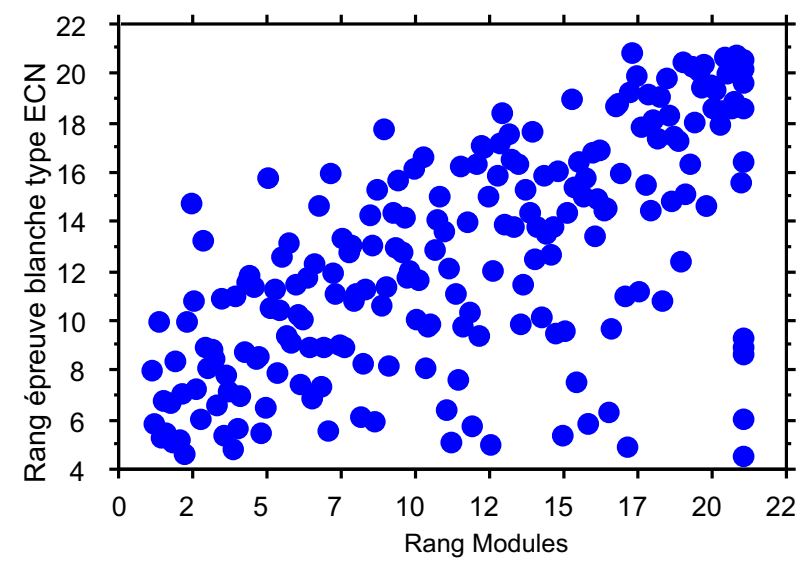

Fig.4. Corrélation entre le rang de classement obtenu aux modules du deuxième cycle et a) le rang de classement aux $E C N$ officielles et b) le rang de classement à l'épreuve blanche de type ECN. ECN : Épreuves Classantes Nationales.

classement pour chacun des dossiers était relativement faible, là où l'on souhaiterait, dans l'idéal, un rang de classement par étudiant. Ainsi, l'étendue la plus importante était observée pour le dossier 9 : étendue des rangs de classement de 96 pour un effectif d'étudiants de 211, témoignant ainsi d'un nombre élevé de rangs ex aequo.

L'étendue des rangs de classements la plus faible était observée pour le dossier 10 (épreuve de LCA) : 69 rangs de classement pour 211 étudiants. De plus, le score obtenu au dossier de LCA n'influençait pas le rang de classement. Ce résultat va à l'encontre des données récentes publiées par Lorette et al. ${ }^{[11]}$. Ces derniers ont en effet démontré que l'épreuve de LCA 
aux ECN de 2009 avait eu un effet significatif sur le rang de classement des étudiants, au même titre qu'un dossier clinique classique. Ainsi, sur 6258 candidats, l'épreuve de LCA avait fait gagner jusqu' à 561 places à un étudiant et perdre jusqu'à 595 à un autre. Les divergences observées entre nos résultats et ces derniers pourraient être dus à la faible valeur discriminante de l'épreuve de LCA proposée lors de l'épreuve blanche de type ECN de l'inter-région Grand-Est de février 2010. De plus, d'une manière plus large, aucun dossier de cette épreuve blanche ne semble avoir influencé de manière franche le classement final des étudiants.

L'ensemble de ces résultats souligne que la fonction classante n'était sans doute pas assurée de manière convenable par l'épreuve blanche de type ECN de l'inter-région Grand-Est. Cette donnée pourrait aller dans le sens des craintes formulées par certains auteurs mettant en doute la validité de l'outil ECN pour classer les étudiants ${ }^{[2,4,5]}$.

Caractère prédictif des résultats de l'épreuve blanche de type ECN de l'inter-région Grand-Est de mars 2010 pour le classement aux ECN officielles de juin 2010

L'objectif des ECN est de classer les étudiants. Sans entrer dans la définition des aptitudes à évaluer pour qualifier de « bon » un étudiant, par ailleurs discutées par certains auteurs ${ }^{[3,5]}$, il est certain que la reproductibilité des résultats, pour un outil d'évaluation choisi, est une qualité essentielle. Le classement des étudiants doit être relativement superposable d'un examen à l'autre, s'il est réalisé selon des modalités similaires et dans un laps de temps court.

Nos résultats mettent en évidence une faible corrélation entre les rangs de classement à l'épreuve blanche de type ECN et aux ECN officielles. La corrélation observée était surtout médiocre pour les étudiants les mieux classés aux ECN officielles, alors qu'elle était meilleure mais encore faible pour les étudiants les plus mal classés.

Nous considérons que les ECN officielles sont le gold standard. Il est donc possible que l'épreuve blanche avait des qualités intrinsèques mauvaises, hypothèse soutenue par les qualités discriminantes médiocres des dossiers avec de nombreux rangs de classement ex aequo. Malgré les précautions prises pour rédiger les dossiers des épreuves blanches de type ECN et les grilles de correction, des écarts de scores conséquents ont été relevés entre le premier et le deuxième correcteur, cet écart de score étant maximal pour le dossier 3. Cet écart de score est surprenant puisque les correcteurs, professeurs des universités, maîtres de conférences des universités et assistants hospitalo-universitaires (AHU) avaient reçu des consignes précises de correction, lors d'un séminaire précédent la remise des copies.

Les ECN officielles mobilisent une organisation rodée et lourde en termes de préparatifs et de corrections réalisées exclusivement par des professeurs des universités, réunis sur des périodes courtes pour des corrections où l'écart de scores entre deux correcteurs ne doit pas excéder cinq points. La faible qualité discriminante de l'épreuve blanche de type ECN de l'inter-région Grand-Est met en exergue la difficulté de mobiliser une telle dynamique et une même organisation au plan local, du fait des moyens financiers moindres disponibles pour ces épreuves blanches mais également du moindre nombre de professeur des universités disponibles pour cette correction et de la difficulté de rassembler une banque de dossiers de type ECN de grande qualité. Ceci explique le choix d'écart de score toléré entre deux correcteurs de 15 points, ce qui, au regard d'une épreuve qui se veut discriminante, est un choix d'écart de scores très large. En effet, la révision des corrections est réduite de manière importante lorsque l'écart de score admis entre deux correcteurs augmente (tel que 15 points pour l'épreuve blanche de type ECN versus cinq seulement pour les ECN officielles). Cela ne favorise pas une grande précision lors de l'élaboration de la grille de correction, ni lors de l'estimation des scores, ce qui peut expliquer la non prédictibilité des rangs de classement de l'épreuve blanche de type ECN. De plus, les AHU ont une expérience en docimologie à acquérir, ce qui peut expliquer un écart de scores important, même en présence d'une grille précise.

S'il est certain, à la vue des ces résultats, que l'épreuve blanche de type ECN qui avait été proposée ne prédisait pas le rang de classement des étudiants, 
les capacités d'amélioration de telles épreuves blanches restent faibles. L'amélioration ne pourra être obtenue que par l' augmentation des valeurs discriminantes des dossiers et de leur grille de correction, ainsi que par la diminution de l'écart toléré de scores entre deux correcteurs.

$\mathrm{Vu}$ le faible caractère prédictif du rang de classement à l'épreuve blanche de type ECN, il pourrait être intéressant, sur un plan local, de chercher si les scores obtenus au cours du deuxième cycle peuvent prédire le rang de classement aux ECN officielles. Cependant, cette hypothèse est mise à mal par nos résultats, du fait de la faible corrélation entre les résultats obtenus durant le deuxième cycle et les ECN officielles ou l'épreuve blanche.

Bien que le caractère prédictif de l'épreuve blanche soit médiocre, le maintien de tels examens blancs pourrait préparer les étudiants aux ECN officielles, si ce n'est en prédisant leur rang de classement, du moins en étant un stimulant pour leurs apprentissages et fournissant aux étudiants une première confrontation avec des conditions proches de celles des ECN officielles. En effet, une étude avait mis en évidence que, parmi les facteurs prédictifs du rang de classement aux ECN, le fait de ne pas avoir participé à une épreuve blanche était associé à un moins bon rang de classement final ${ }^{[14]}$. Les épreuves de type ECN pourraient donc constituer une aide dans la préparation aux ECN même si ce n'est pas en raison de leur capacité à prédire les résultats aux ECN officielles.

Une limite à nos résultats est le délai de quatre mois entre l'épreuve blanche de type ECN et les ECN officielles. Certains étudiants auraient pu fournir davantage d'efforts d'apprentissage que d'autres pendant cette période, avec pour conséquence des modifications dans le classement final. $\mathrm{Si}$ la préparation aux ECN débute dès le début du deuxième cycle des études médicales, c'est-à-dire très en amont des ECN officielles, ce laps de temps plus court de quatre mois peut néanmoins être mis à profit par certains étudiants selon les efforts finaux qu'ils fournissent.

En conclusion, notre étude a démontré que les qualités discriminantes de l'épreuve blanche de type ECN de l'inter-région Grand-Est étaient faibles. La corrélation entre cette épreuve blanche et les ECN officielles était médiocre. Il n'était donc pas possible de prédire le classement aux ECN officielles sur la base de l'épreuve blanche type ECN de l'inter-région Grand-Est. Cependant, ces résultats mettent en lumière la difficulté d'établir une épreuve blanche dans les conditions identiques à celles des ECN officielles, notamment du fait des moyens matériels et humains moindres sur un plan local que pour les ECN officielles nationales

\section{Contributions}

Rachel Vieux a assuré la conception du protocole de recherche, le recueil des données, l'analyse statistique, l'interprétation des résultats et l'écriture du manuscrit. Yannick Béjot a contribué à l'interprétation des résultats et à l'écriture du manuscrit. Marc Braun a contribué à la conception de la problématique de la recherche et à la relecture du manuscrit. François Kohler a contribué à la conception du protocole de recherche, a fourni une aide à l'analyse statistique et à la relecture du manuscrit.

\section{Références}

1. Evidence-based Medicine working group. Evidencebased Medicine: a new approach to teaching the practice of Medicine. JAMA 1992;268:2420-5.

2. Jouquan J, Honnorat C. Que reste-t-il comme vertus aux épreuves classantes nationales ? Pédagogie Médicale 2006;7:197-200.

3. Roland J. L'éducation médicale vue par un responsable institutionnel. Pédagogie Médicale 2006;7:233-7.

4. Roussel F. Épreuves classantes nationales, une inadéquation majeure entre le nombre des postes. Presse Med 2006;35:437-8.

5. Roussel F. Pour une transformation des épreuves classantes nationales en un examen classant national. Pédagogie Médicale 2006;7:228-32.

6. Duhaut P. L'examen national classant (ENC) quatre années après sa mise en place : qu'en penser ? Rev Med Int 2008;29:531-4. 
7. Durieux P. La lecture critique n'est pas destinée à des universitaires déconnectés de la réalité. Presse Med 2009;38:1006-7.

8. Fagniez PL. Evaluation de la mise en place de l'enseignement de la lecture critique d'article dans les Facultés de Médecine et opportunité de son intégration aux Épreuves classantes nationales. Rapport de mission au Ministre délégué à l'Enseignement supérieur et à la Recherche François Goulard 2007. http://media.enseignementsuprecherche.gouv.fr/file/08/7/20087.pdf.

9. Jouquan J. La lecture critique d'article scientifique audelà du contexte franco-français. Pour éviter que l'arbre ne cache la forêt ! Pedagogie Médicale 2009; 10:77-82.

10. Lorette G. La lecture critique d'article fait partie des disciplines fondamentales. Presse Med 2009;38:1007.

11. Lorette G, Jolly D, Ambrosi P, Dreyfuss D, Magne JL, Kohler C, Duhaut P, Gérard JL. Influence de la lecture critique d'articles sur les classements des étudiants aux épreuves classantes nationales 2009 Presse Med 2010;39:118-25.

12. Steichen O, Grateau G. Le zen et l'art de la lecture critique d'article. Presse Med 2009;38:1005-6.

13. Ricketts C, Archer J. Are national qualifying examinations a fair way to rank medical students? Yes. BMJ 2008;337:1282.

14. Andujar P, Bastuji-Garin S, Botterel F, Prevel M, Farcet JP, Claudepierre P. Facteurs associés à la performance des étudiants à l'Examen Classant National dans une faculté de médecine française. Presse Med 2010;39:e134-40.

Correspondance et offprints : Rachel Vieux, Service de Réanimation Néonatale, Soins Intensifs et Médecine Néonatale, Maternité Régionale A. Pinard, 10 rue du Docteur Heydenreich, 54042 Nancy Cedex, France.

Email : r.vieux@maternite.chu-nancy.fr 